\title{
Entropy Optimized Palmprint Enhancement Using Genetic Algorithm and Histogram Equalization
}

\author{
S. Palanikumar ${ }^{1, *}$, M. Sasikumar ${ }^{2}$, J. Rajeesh $^{3}$ \\ ${ }^{1}$ Anna University,Trichy and Asst.Professor,Dept. of IT, Noorul Islam Centre for Higher Education,Noorul Islam University, Kumaracoil, \\ India \\ ${ }^{2}$ Dept. of IT, Noorul Islam Centre for Higher Education, Noorul Islam UniversityKumaracoil, India \\ ${ }^{3}$ Anna University, Trichy and Senior Lecturer,Dept. of ECE and, Noorul Islam Centre for Higher Education, Noorul Islam University, \\ Kumaracoil, India \\ palanikumarcsc@yahoo.com,drmsasikumar@yahoo.com,rajeesh_j@yahoo.co.in
}

\begin{abstract}
Palmprint identification is a subcategory of biometrics identification, which can be efficiently used to identify the people. Palmprint-based identification is currently a potential alternative to human identification method of a well known fingerprint-based identification. In order to achieve high identification accuracy, all components of the scanned palmprint image need to be enhanced, i.e. palmprint lines, textures and hand geometry features. Based on Histogram Equalization (HE), a contrast enhancement scheme named Adaptively Increasing Value Histogram Equalization (AIVHE) can be used as an enhancement technique. In AIVHE method, the enhancement is controlled by two user parameters beta and gamma. Furthermore, AIVHE method enhances the contrast but the detailed information in the palmprint is not preserved. In order to enhance the palmprint and preserve the information, Genetic Algorithm (GA) is used to optimize the value of beta and gamma based on entropy value. The experiments results show that the proposed method does enhancement and brightness preservation and thereby improves information in the palmprint. Entropy of images is used as a fitness function in this work.
\end{abstract}

Keywords Enhancement, Genetic Algorithm, Histogram, Palmprint

\section{Introduction}

Contrast enhancement is a display technology that improves the exhibition effect by increasing the dynamic range of gray intensity of the input image. It is an important technology for the improvement of digital image quality. Contrast enhancement techniques are classified into two categories. They are spatial domain methods and Frequency domain methods. Spatial domain methods perform the enhancement on the pixels of image itself. In Spatial Domain Methods, there are number of contrast enhancement techniques like Image Negatives, Contrast Stretching, Power-law Transforms, Gray-level Slicing, Bit-plane Slicing, Histogram Equalization, Histogram Specification, and Histogram Modification. Frequency domain methods perform the enhancement by taking the transform of the input image like Fourier Transform, Fast Fourier Transform, etc. Some of the frequency domain methods are low-pass filtering, high-pass filtering, band-pass filtering, unsharp masking, etc. Curvelet Based Palmprint Enhancement (CBPE) method S.Palaniku mar et al.[1] perform enhancement in the presence of noise.

* Corresponding author:

palanikumarcsc@yahoo.com (S.Palanikumar)

Published online at http://journal.sapub.org/ijge

Copyright (C) 2012 Scientific \& Academic Publishing. All Rights Reserved
But it does not improve the contrast. It is necessary to develop a better enhancement technique particularly for palmprint images so that palmprint identification accuracy can be improved further.

\subsection{Global Histogram Methods}

Global histogram equalization uses the histogram information of the whole input image as its transformation function. This transformation function stretches the contrast of the high histogram region and compresses the contrast of the low histogram region. This global histogram equalization method is simple and powerful, but it cannot adapt to local brightness features of the input image. This fact limits the contrast stretching ratio and causes significant contrast losses in the background and other small regions.

\subsection{Local Histogram Methods}

To overcome the limitations of global histogram equalization, a local histogram equalization method has been developed, which can also be termed block-overlapped histogram equalization. This method allows each pixel to adapt to its neighboring region, so that high contrast can be obtained for all locations in the image. Local histogram equalization must be performed for all pixels in the entire image frame. The computational complexity is very high. Local Contrast Palmprint Enhancement (LCPE) method S.Palanikumar et al.[2] provides better contrast enhancement. But the com- 
putation cost is high.

\section{Traditional Histogram Equalization Method}

HE obtains input-output transfer function by means of the histogram of input images. The probability density function (PDF), $\mathrm{P}(\mathrm{k})$ of the image is defined as follows,

$$
\mathrm{P}(\mathrm{k})=\frac{\mathrm{n}_{\mathrm{k}}}{\mathrm{N}} \quad \text { for } \mathrm{k}=0,1,2 \ldots . \mathrm{L}-1
$$

Where $\mathrm{k}-\mathrm{k}^{\text {th }}$ gray level of pixel , $\mathrm{n}_{\mathrm{k}}-$ no. of pixels in $\mathrm{k}^{\text {th }}$ gray level, $\mathrm{N}-$ Total no. of pixels of an image, $\mathrm{L}-1$ Maximum gray level.

And cumulative density function CDF is defined as,

$$
\mathrm{C}(\mathrm{k})=\sum_{\mathrm{i}=0}^{\mathrm{k}} \mathrm{P}(\mathrm{i}) \quad \text { for } \mathrm{k}=0,1, \ldots . \mathrm{L}-1
$$

And input-output transfer function is

$$
\mathrm{f}(\mathrm{x})=\mathrm{X}_{0}+\left(\mathrm{X}_{\mathrm{L}-1}+\mathrm{X}_{0}\right) * \mathrm{C}(\mathrm{x})
$$

HE can not control the effect of enhancement. While histogram is centralized in a narrow area, the method tends to produce undesirable artefacts and occurrence of noise.

Nicholas Sia Pik Kong et al.[3] proposed multiple layers block overlapped histogram equalization for local content emphasis. This method consists of three stages, which are enhancement stage, noise reduction stage and merging stage. First, it generates three versions of the input image with three different window sizes. Second, these three outputs are filtered with median filter. Finally, these filtered three outputs are merged as single output image. This method does not need predefined parameters. Moreover, It reveals hidden details in the image.

R. Crane[4] Histogram Stretching (HS) and H-C. Kim et al.[5] Improved Histogram Stretching IHS are applied to stretch the histogram of image to full dynamic range and suitable to use on centralized histogram of image. Since both methods work on gray levels to calculate input-output mapping curve, and hence they are sensitive to the noise influence.

The localized contrast manipulation method Z. Yu et al.[6] achieves the enhancement by analysing local statistical information, and then obtaining the function to transfer the effect of images. The method of Automatic Video Contrast Enhancement K.H. Goh et al.[7] performs histogram analysis by computing some statistical information of the image to select the mapping function for enhancing. Both methods achieve contrast enhancement from the view point of characters of image distribution. The lack of both the methods is that they can not provide the full variety of input/output transfer function.

R. C. Gonzalez et al.[8] discuss about traditional histogram equalization. Global Enhancement methods are relatively simple than other methods. The weakness of these methods is that they can not provide precise image contrast enhancement effect because the inflexibility of these meth- ods and the inability of noise elimination.

\subsection{Histogram Equalization Based Methods}

The method performs the remapping in the gray levels to produce uniform distribution in the order of input images. But it is also being criticized that it always causes unacceptable visual artifacts. There are many solutions been proposed to conquer the weakness of the traditional $\mathrm{HE}$ method[9-14]. The three most famous adaptive histogram equalization methods are Improving $\mathrm{HE}$ methods, Spatial processing $\mathrm{HE}$ methods, and Probability density function (PDF) shaping HE methods. Some of the PDF shaping methods are,

\subsubsection{Bin Underflow and Bin Overflow (BUBO)}

To improve the undesirable artifacts caused by traditional HE method, the S. Yang et al.[13] BUBO method puts constraints to avoid over enhancement, where $C_{B U}$ and $C_{B O}$ are bin underflow and bin overflow thresholds. BUBO is an effective technique for contrast enhancement but in some cases the method still can not expand gray level distribution to expand dynamic range of input image. Although we can add the function of adjusting parameter into BUBO to expand image for more dynamic range the method still need to adjust different parameter for input images. To overcome the drawbacks of the Traditional HE Methods, the BUBO method puts constraint avoid over enhancement by,

$$
\begin{gathered}
\mathrm{P}_{\mathrm{BUB} 0}(\mathrm{k})=\left\{\begin{array}{ccc}
C_{B O} & \text {,if } & P(k)>C_{B O} \\
P(k) & \text {,if } & C_{B U} \geq P(k) \leq C_{B O} \\
C_{B U} & \text {,if } & P(k)<C_{B U}
\end{array}\right. \\
\mathrm{C}_{\mathrm{BU}}=(1-\alpha) / \mathrm{N} \\
\mathrm{C}_{\mathrm{BO}}=(1+\alpha) / \mathrm{N}
\end{gathered}
$$

Where $\alpha$ varies from 0 to infinity, and $\mathrm{N}$ is the total number of pixels in the given input image. It can't expand grey-level distribution to expand dynamic range of input image. To expand image for more dynamic range the method still need and adjust different parameter for input images.

The former puts constraint on the PDF with the bin underflow and bin overflow thresholds to prevent a significant change of gray levels. In H-J Kim et al.[14], the AMHE method scales the original shape of the PDF based on the middle value from the maximum and minimum values with original image. For providing a well designed adaptive contrast enhancement effectively and prevent a significant change of gray levels, this study focuses on the PDF shaping HE techniques to propose a simple contrast enhancement scheme Ching-Hsi Lu et al.[15], named AIVHE to enhance the contrast in a more efficient way.

Hashemi et al.[16] uses a simple and novel chromosome representation together with corresponding operators. This method makes natural looking images especially when the dynamic range of input image is high. Termination is based the difference of best fitness in two last consecutive generations or the maximum number of generations specified by the user. Changjiang Zhang et al.[17] presented a enhance- 
ment algorithm for typhoon cloud image based on combining discrete undecimated wavelet transform (UWT) with Genetic Algorithm(GA).

Om Prakash Verma et al.[18] uses fuzzy logic and modified artificial ant colony system techniques for enhancement of high dynamic range color images. C. Munteanu et al.[19] introduces a new automatic image enhancement technique based on real-coded GA. Omid Khayat et al.[20] presented an algorithm for image enhancement. Here, parametric indices of fuzziness(PIF) is introduced, which servers as the optimization criterion of the contrast enhancement procedure.

Mark'us Gud mundsson et al.[21] developed an algorithm that detects well-localized, unfragmented, thin edges in medical images based on optimization of edge configurations using a GA. Nemanja I. Petrovic et al.[22] presented a novel method for impulse noise filter construction, based on the switching scheme with two cascaded detectors and two corresponding estimators. Genetic programming as a supervised learning algorithm is employed for building two detectors with complementary characteristics.

Sara Hashemi et al.[23] presented a novel and efficient contrast enhancement method based on GA. This method was stronger than counterpart methods in terms of contrast and detail enhancement and producing natural looking images. C.J. Zhang and X.D. Wang[24] presented an efficient de-noising and enhancement algorithm for typhoon cloud image .GA and non-linear gain operator are used to modify the stationary wavelet coefficients at coarse resolution levels in order to enhance the details of a typhoon cloud image. Xia Fei and Ye Qing[25] worked with GA for enhancing the misty images. The method seeks the threshold value using genetic algorithm which segment the establishing shot and the close shot.

$\mathrm{Mu}$ Dongzhou et al.[26] combines the differential evolution and GA in the image enhancement process and utilize the quickly searching ability of the algorithm to carry out the adaptive mutation and searches. Sandra Sovilj-Nikic[27] presented partition based median filtering using genetic algorithm. Mantas Paulinas and Andrius Ušinskas[28] emphasis that GA are the most powerful unbiased optimization techniques for sampling a large solution space.

Here,[17-27] uses the genetic algorithm to optimize parameters in enhancement algorithm or perform enhancement in genetic domain. Furthermore, it improves the contrast and detail preservation with natural looking. This paper apply GA in AIVHE method.

\subsubsection{AIVHE Contrast Enhancement Method}

The method reshapes the original PDF to obtain new PDF to prevent a significant change in the gray levels. It also provides a mechanism of adjustment to contrast enhancement by means of adaptive constraint parameter $\alpha(k)$ for adjustment automatically, which is determined by the initial value $\gamma$ and user defined parameter $\beta$. AIVHE divides the original PDF into upper and lower blocks on the basis of $\mathrm{P}_{\text {bas. }}$. A value of maximum threshold $\mathrm{P}_{\mathrm{h}}$ is then be set to restrict the variation of the $\mathrm{P}_{\mathrm{AIVHE}}(\mathrm{k})$, and then limit the value of $\mathrm{P}_{\mathrm{AIVHE}}$ (k) be not greater than $P_{h}$. AIVHE reshapes original PDF and obtain the $\mathrm{P}_{\text {AIVHE }}(\mathrm{k})$ by,

$$
\mathrm{P}_{\text {AIVHE }} \quad(\mathrm{k})=\left\{\begin{array}{ccc}
P_{h} & \text { if } & P(k) \geq P_{h} \\
P(k)-\alpha(k)\left(P(k)-P_{\text {bas }}\right) \times \beta, & \text { if } & P_{\text {bas }}<P(k)<P_{h}(5) \\
P(k)+\alpha(k)\left(P_{\text {bas }}-P(k)\right) \times \beta, & \text { if } & P(k) \leq P_{\text {bas }}
\end{array}\right.
$$

Where $\mathrm{P}_{\text {bas }}$ is the average PDF, $\mathrm{P}_{\mathrm{h}}$ - is double of $\mathrm{P}_{\text {bas }}, \beta$ To adjust the enhance effect by user, and $\alpha(k)$ - automatic adjusted adaptive constraint parameter. The initial value for $\beta$ is a real number in the range of $[0,1]$. The function of $\mathrm{HE}$ is produced when $\beta$ is set to zero and $\mathrm{P}_{\text {bas }}$ is the mean value of the maximum and minimum value of $\mathrm{P}(\mathrm{k})$. Dark and bright regions stretching is controlled by $\gamma$ at $\alpha(k)$. Whole contrast enhancement effect of the image by is obtained by $\beta$. The effective constraint parameter can be calculated as

$$
\begin{gathered}
\alpha(\mathrm{k})=\left(1-\left(\mathrm{X}_{\mathrm{m}}-\mathrm{k}\right) / \mathrm{X}_{\mathrm{m}}\right)^{2} \times(1-\gamma)+\gamma \text { if }, \mathrm{k} \leq \mathrm{X}_{\mathrm{m}} \\
\left(1-\left(\mathrm{k}-\mathrm{X}_{\mathrm{m}}\right) /\left((\mathrm{L}-1)-\mathrm{X}_{\mathrm{m}}\right)\right)^{2} \times(1-\gamma)+\gamma \text { if, } \mathrm{k}>\mathrm{X}_{\mathrm{m}}
\end{gathered}
$$

Where, $\mathrm{X}_{m}$ - Mean brightness to increment the mean value of brightness gradually, $\gamma$ - The initial value is a real number in the range of $[0,1]$. It decides the distribution of pixels in dark and bright region. By the $\mathrm{P}_{\text {AIVHE }}(\mathrm{k})$, the cumulative distributive function, $\mathrm{C}_{\mathrm{AIVHE}}(\mathrm{k})$, is straight forward accumulated. $\mathrm{C}_{\text {AIVHE }}(\mathrm{k})$ is normalized to gray level[0, L-1] and then the output image is as follows,

$$
\mathrm{f}(\mathrm{k})=(\mathrm{L}-1) \times\left(\mathrm{C}_{\text {AIVHE }}(\mathrm{k})\right) /\left(\mathrm{C}_{\text {AIVHE }}(\mathrm{L}-1)\right)
$$

Where $f(k)$ - input/output transfer function, L-1 - maximum gray level, $\mathrm{k}-\mathrm{k}^{\text {th }}$ gray level, $\mathrm{C}_{\text {AIVHE }}(\mathrm{k})-$ cumulative density function.

\section{Entropy Optimized Palmprint Enhancement (EOPE)}

In AIVHE method, there are two user controlled parameters namely $\beta$ and $\gamma$. These two parameters control the enhancement. Moreover, using AVIHE method the contrast is enhanced while the brightness is not preserved . Since the parameters are user controlled, that leads to ineffective enhancement. The proposed algorithm is based on GA which optimizes the value of $\beta$ and $\gamma$ based on the fitness function entropy and hence preserve the brightness.

\subsection{Genetic Algorithm}

Genetic Algorithms are search algorithms based on the mechanics of the natural selection process (biological evolution). The most basic concept is that the strong tend to adapt and survive while the weak tend to die out. That is, optimization is based on evolution, and the survival of the fittest concept. GAs have the ability to create an initial 
population of feasible solutions, and then recombine them in a way to guide their search to only the most promising areas of the state space. Each feasible solution is encoded as a chromosome (string) also called a genotype, and each chromosome is given a measure of fitness via a fitness (evaluation or objective) function. The fitness of a chromosome determines its ability to survive and produce offspring.

A finite population of chromosomes is maintained. GAs use probabilistic rules to evolve a population from one generation to the next. The generations of the new solutions are developed by genetic recombination operators such as reproduction, crossover and mutation.

Reproduction means selecting the fittest to reproduce. Crossover means combining parent chromosomes to produce children chromosomes. Cross over combines the fittest chromosomes and passes superior genes to the next generations. Mutation means altering some genes in a chromosome. Mutation ensures the entire state-space will be searched and can lead to population out of a local minima.

The process of GA follows this pattern.

1) Create an initial population (usually randomly generated string).

2) Evaluate all of the individuals (apply some function or formula to the individuals)

3) Select a new population from the old population based on the fitness of the individuals as given by the evaluation function.

4) Apply some genetic operators (mutation \& crossover) to members of the population to create new solutions.

5) Evaluate these newly created individuals.

6) Repeat steps 3-6 (one generation) until the termination criteria has been satisfied (usually perform for a certain fixed number of generations).

It is very important to define a good fitness function that rewards the right kinds of individuals. The proposed algorithm uses entropy as the fitness function

$$
\text { Fitness }=\text { Entropy measure }
$$

\section{Results and Discussion}

\subsection{Quality Measurements}

Here the enhancement level of proposed method is compared with that of Histogram Equalization, BUBO, AIVHE method using two quality measurements, Absolute Mean Brightness Error (AMBE) and Entropy.

\subsubsection{AMBE}

AMBE is the difference between the Global Mean of input image to the Global Mean of the output image.

$$
\mathrm{AMBE}=\mathrm{abs}((\text { Mean of input image })-(\text { Mean of output image })
$$

\subsubsection{Entropy}

It is defined as a measure of the average information content of an Image an. It is calculated using the formula

$$
\mathrm{E}=-\sum \mathrm{P}\left(\mathrm{x}_{\mathrm{i}}\right) * \log _{2} \mathrm{P}\left(\mathrm{x}_{\mathrm{i}}\right)
$$

Where $\mathrm{P}\left(\mathrm{x}_{\mathrm{i}}\right)$ is the probability of the $\mathrm{i}^{\text {th }}$ gray level.

The input palms are acquired by using Nokia 2700 with resolution of $1200 \times 1700$. Experiments are done with palmprint image of size $256 \times 256$.The simulation is done in Matlab R2008a with 100 test images.

The observations show that the proposed algorithm enhances the contrast of the image and also preserve the brightness. The original image and output from different enhancement methods are given in Fig 1 and Fig. 4. Fig. 2 and Fig. 5 shows how the contrast of the palmprint is enhanced. From Fig.3 and Fig.6, it is clear that the brightness of the palmprint image is well preserved.

Table 1 gives the comparison of entropy of the proposed method with other methods. The entropy of the EOPE method is higher than that of all other methods. Hence, this method gives enhancement of palmprint with more information.

Table 2 gives the comparison of AMBE of the proposed method with other methods. The proposed method gives lesser AMBE value than the other methods. The AMBE of the EOPE method is lesser that of all other methods. Hence, this method gives enhancement of palmprint while preserving the brightness.

Fig. 7 and Fig. 8 shows the comparison chart of entropy value and AMBE of different methods. From Fig.7 ,EOPE technique has the higher entropy value. By observing the results of Fig. 8, the EOPE technique has lowest AMBE value. So this method has more information and preserves more brightness than BUBO ,HE and AIVHE method.

The initial population for GA is kept as 20 and the generations are limited to 20 .

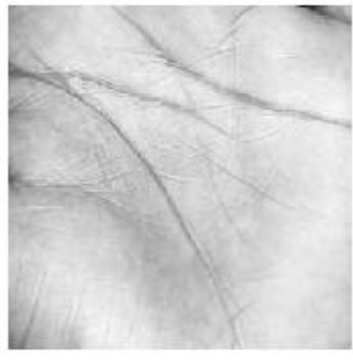

(a)

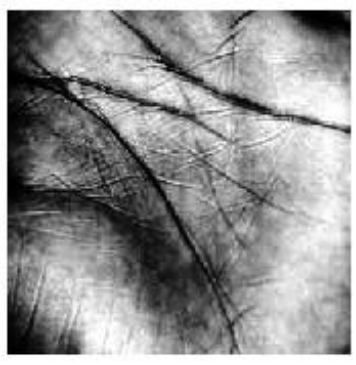

(c)

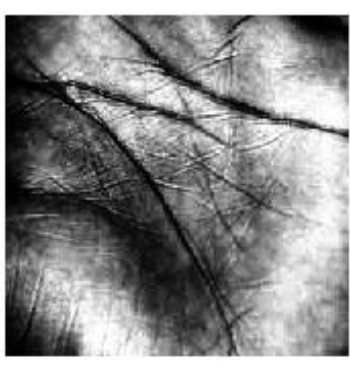

(b)

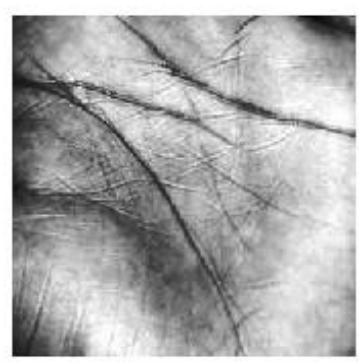

(d) 


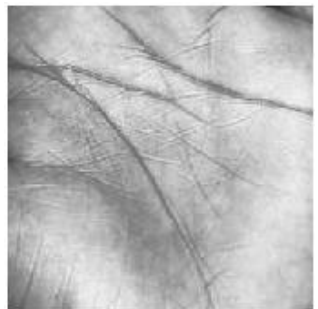

(e)

Figure 1. (a) original image (b) enhanced by HE (c) enhanced by BUBO (d) enhanced by AIVHE $\gamma=0.35, \beta=0.35$ (e) enhanced by EOPE
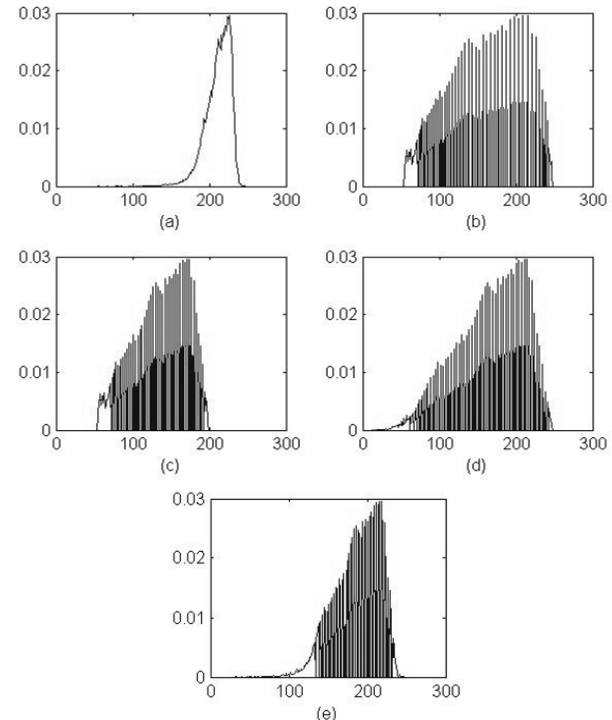

Figure 2. Histograms of figure 1. (a) original image (b) enhanced by HE (c) enhanced by BUBO (d) enhanced by AIVHE $\gamma=0.35, \beta=0.35$ (e) enhanced by EOPE

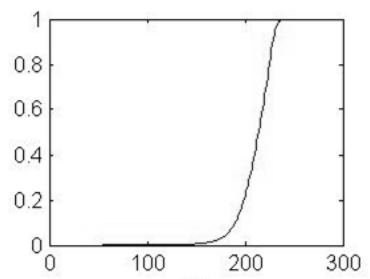

(a)
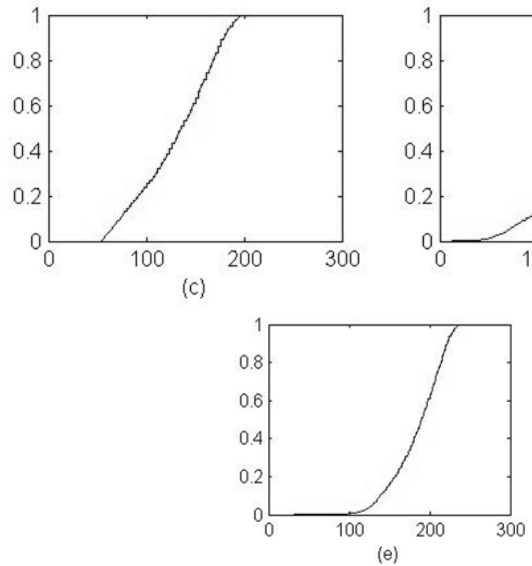

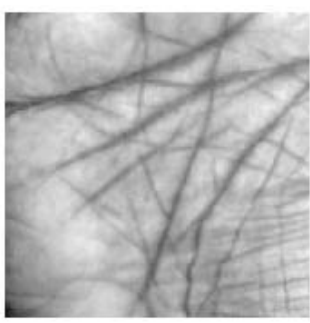

(a)

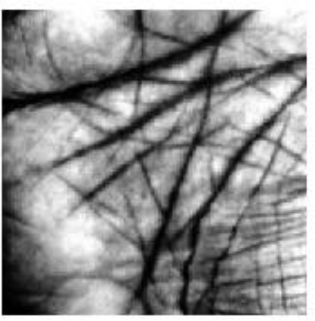

(c)

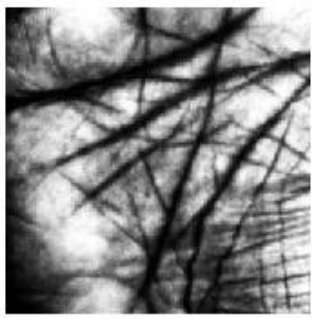

(b)

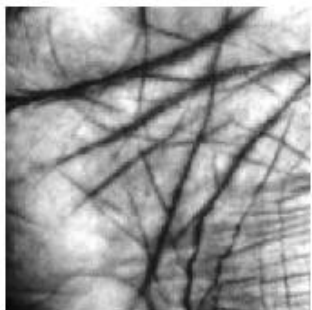

(d)

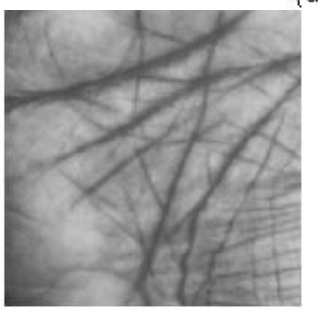

(e)

Figure 4. (a) original image (b) enhanced by HE (c) enhanced by BUBO (d) enhanced by AIVHE $\gamma=0.35, \beta=0.35$ (e) enhanced by EOPE
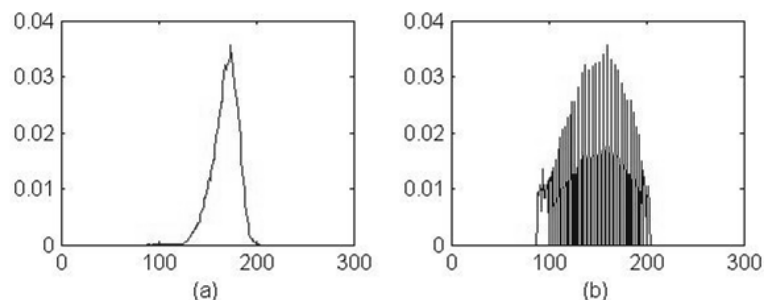

(b)
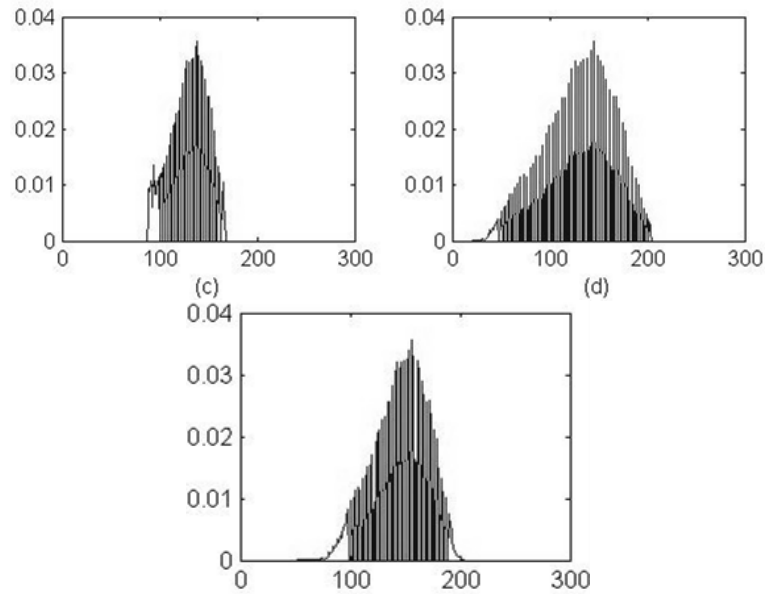

(e)

Figure 5. Histograms of figure 4. (a) original image (b) enhanced by HE (c) enhanced by BUBO (d) enhanced by AIVHE $\gamma=0.35, \beta=0.35$ (e) enhanced by EOPE
Figure 3. CDF of figure 1. (a) original image (b) enhanced by HE (c) enhanced by BUBO (d) enhanced by AIVHE $\gamma=0.35, \beta=0.35$ (e) enhanced by EOPE

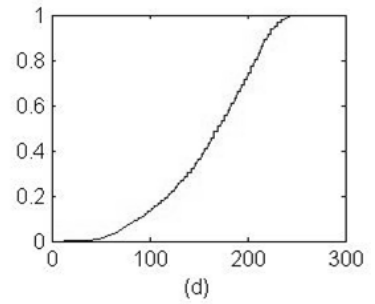




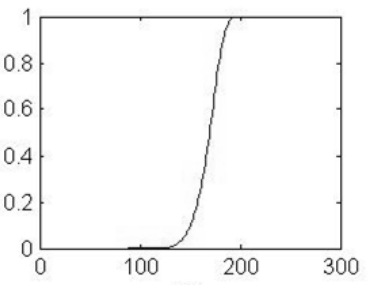

(a)

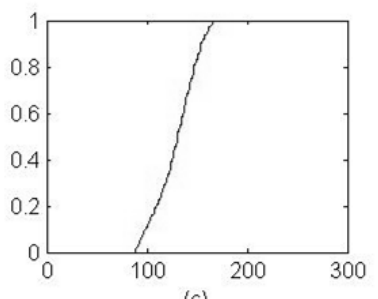

(c)

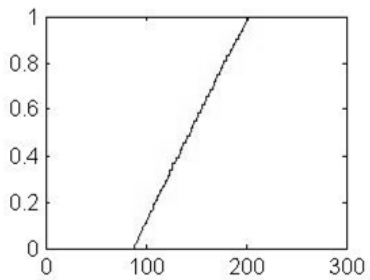

(b)

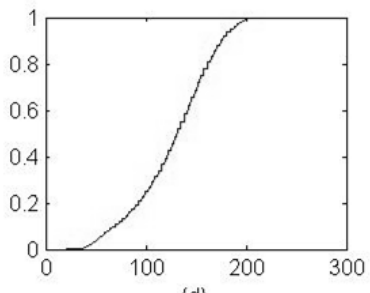

(d)

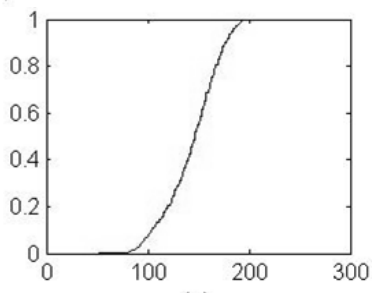

(e)

Figure 6. $\mathrm{CDF}$ of figure 4. (a) original image (b) enhanced by HE (c) enhanced by BUBO (d) enhanced by AIVHE $\gamma=0.35, \beta=0.35$ (e) enhanced by EOPE

Table 1. Entropy

\begin{tabular}{|c|c|c|c|c|c|}
\hline Entropy & Palm1 & Palm2 & Palm3 & Palm4 & Palm5 \\
\hline HE Method & 5.7810 & 5.5429 & 5.8498 & 5.4927 & 5.6188 \\
\hline $\begin{array}{c}\text { BUBO } \\
\text { Method }\end{array}$ & 5.7799 & 5.5429 & 5.8498 & 5.4966 & 5.6188 \\
\hline $\begin{array}{c}\text { AIVHE } \\
\text { Method }\end{array}$ & 5.8696 & 5.6554 & 5.9146 & 5.6672 & 5.7696 \\
\hline $\begin{array}{c}\text { GA-AIVHE } \\
\text { method }\end{array}$ & 5.8847 & 5.6699 & 5.9300 & 5.6829 & 5.8037 \\
\hline
\end{tabular}

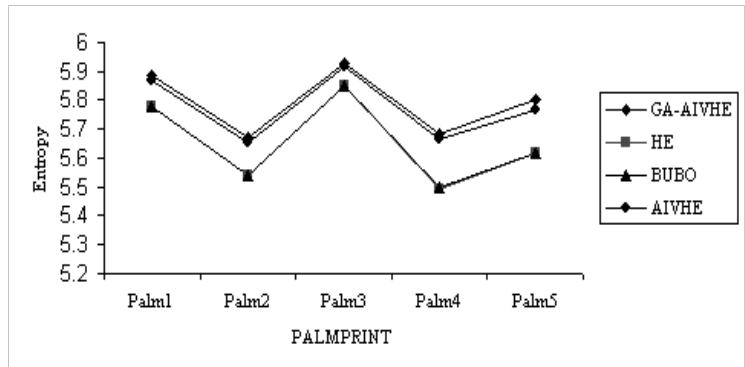

Figure 7. Enhancement Methods Vs Entropy

Table 2. AMBE

\begin{tabular}{|c|c|c|c|c|c|}
\hline AMBE & Palm1 & Palm2 & Palm3 & Palm4 & Palm5 \\
\hline HE Method & 62.20 & 23.04 & 46.547 & 17.40 & 10.40 \\
\hline $\begin{array}{c}\text { BUBO } \\
\text { Method }\end{array}$ & 79.44 & 38.99 & 66.61 & 31.238 & 25.54 \\
\hline $\begin{array}{c}\text { AIVHE } \\
\text { Method }\end{array}$ & 48.27 & 40.726 & 53.60 & 44.44 & 28.44 \\
\hline $\begin{array}{c}\text { GA-AIVHE } \\
\text { method }\end{array}$ & 25.85 & 24.13 & 30.82 & 19.13 & 21.15 \\
\hline
\end{tabular}

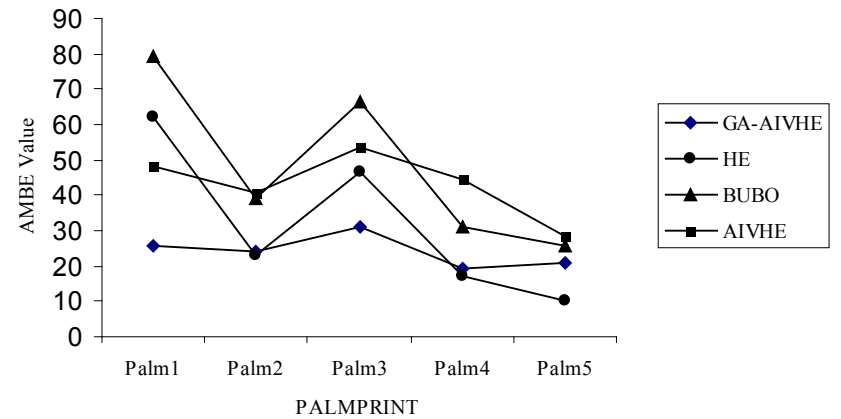

Figure 8. Enhancement Methods Vs AMBE

\section{Conclusions}

The EOPE method for image contrast enhancement enhances image quality. The simulation results show that the method can enhance image contrast effectively by improving the information and preserving the brightness. The GA provide optimum value for $\beta$ and $\gamma$ based on the fitness value.

\section{REFERENCES}

[1] S.Palanikumar, M.Sasikumar, J.Rajeesh ,"Curvelet Based Palmprint Enhancement ", Proceedings of the International Conference on Computing Technologies ICONCT 2009 pp.79-84.

[2] S.Palanikumar, M.Sasikumar, J.Rajeesh "Local Contrast Palmprint Enhancement", UPA International Journal of Image processing and Networking Techniques Vol.3 No.1, June 2010. pp.44-53

[3] Nicholas Sia Pik Kong, Haidi Ibrahim, " Multiple layers block overlapped histogram equalization for local content emphasis", Computers and Electrical Engineering xxx (2010) $\mathrm{xxx}-\mathrm{xxx}$.

[4] R. Crane, Simplified Approach to Image Processing, Prentice-Hall, 1994.

[5] H-C. Kim, B-H. Kwon and M-R. Choi, "An Image Interpolator With Image Improvement For LCD Controller," IEEE Trans. on Electronics, Vol.47, May. 2001,pp.263-271.

[6] Z. Yu and C. Bajaj, "A Fast and Adaptive Method for Image Contrast Enhancement", in 2004 Proc. of ICIP, Vol.2, Oct. 2004, pp. 1001-1004,

[7] K.H. Goh, Y. Huang and L. Hui, "Automatic Video Contrast Enhancement", IEEE Int. Symposium on Consumer Electronics, Sept. 2004, pp. 359- 364.

[8] R. C. Gonzalez, R. E. Woods, Digital image processing, Prentice-Hall, Inc., 2001.

[9] Y. T. Kim, "Contrast enhancement using brightness preserving bihistogram equalization," IEEE Trans. on Consumer Electronics, Vol.43, Feb. 1997, No.1, pp.1-8.

[10] S-D Chen, and A. R. Ramli, "Contrast enhancement using recursive mean-separate histogram equalization for scalable 
brightness preservation", IEEE Trans. Consumer Electronics, vol. 49, no. 4, Nov. 2003, pp. 1301-1309.

[11] K. Zuiderveld, "Contrast Limited Adaptive Histogram Equalization," in Chapter VIII.5, Graphics Gems IV, Cambridge, MA, Academic Press, 1994, pp.474-485.

[12] J-Y Kim, L-S. Kim, and S-H. Hwang, "An Advanced Contrast Enhancement Using Partially Overlapped Sub-block Histogram Equalization", IEEE Trans. on Circuits and Systems for Video Technology, Vol.11, No.4, April. 2001, pp.475-484.

[13] S. Yang, J. Oh, and Y. Park, "Contrast Enhancement Using Histogram with Bin Underflow and Bin Overflow", in 2003 Proc. of ICIP, Vol. 1, Sep. 2003, pp.881 - 884.

[14] H-J Kim, J-M Lee, J-A Lee, S-G Oh, W-Y Kim, "Contrast Enhancement Using Adaptively Modified Histogram Equalization", Lecture Notes in Computer Science, Vol.4319, Dec. 2006, pp. $1150-1158$.

[15] Ching-Hsi Lu, Hong-Yang Hsu and Lei Wang,"A New Contrast Enhancement Technique by Adaptively Increasing the Value of Histogram", IEEE 2009.

[16] Sara Hashemi, Soheila Kiani, Navid Noroozi, Mohsen Ebrahimi Moghaddam, "An image contrast enhancement method based on genetic algorithm', Pattern Recognition Letters Volume 31, Issue 13, October 2010. pp.1816-1824.

[17] Om Prakash Verma, Puneet Kumar, Madasu Hanmandlu, Sidharth Chhabra," High dynamic range optimal fuzzy color image enhancement using artificial ant colony system", Applied Soft Computing, Volume 12, Issue 1,January 2012,pp. 394-404.

[18] C. Munteanu, A. Rosa, "Towards automatic image enhancement using genetic algorithms" Proceedings of the congress on evolutionary computation,2000.

[19] Omid Khayat, Javad Razjouyan, Mina Aghvami, Hamid Reza shahdoosti,babak Loni, "An automated GA-based fuzzy image enhancement method", IEEE Symposium on Computational Intelligence for Image Processing, CIIP '09, May 2009 ,pp. 14-19.
[20] Mark'us Gud mundsson, Essam A. El-Kwae, and Mansur R. Kabuka, "Edge detection in medical images using a genetic algorithm", IEEE Transactions on Medical Imaging, Vol. 17, No. 3, June 1998.

[21] Nemanja I. Petrovic' and Vladimir Crnojevic', Member, IEEE, "Universal impulse noise filter based on genetic programming", IEEE Transactions on Image Processing, Vol. 17, No. 7, July 2008.

[22] Sara Hashemi, Soheila Kiani, Navid Noroozi, Mohsen Ebrahimi Moghaddam, "An image enhancement method based on genetic algorithm", International Conference on Digital Image Processing, 2009 IEEE, Page(s): 167 - 171, DOI 10.1109/ICDIP.

[23] C.J. Zhang X.D. Wang "Typhoon cloud image enhancement and reducing speckle with genetic algorithm in stationary wavelet domain", IET Image Process., 2009, Vol. 3, Issue. 4, pp. 200-216, doi: 10.1049/iet-ipr.2008.0044.

[24] Hong Xia Fei , Ye Qing, "A new threshold segmentation method based on the genetic algorithm for enhancing the images", International Conference on Intelligent System Design and Engineering Application, 2010 IEEE, DOI 10.1109/ISDEA.2010.89.

[25] Mu Dongzhou, Xu Chao and Ge Hongmei "Hybrid genetic algorithm based image enhancement technology", 2011 IEEE.

[26] K. Sri Rama Krishna , A. Guruva Reddy, M.N.Giri Prasad, K.Chandrabushan Rao , M. Madhavi ,"Genetic algorithm processor for image noise filtering using evolvable hardware", International Journal of Image Processing, Volume (4): Issue (3),pp.240-250.

[27] Sandra Sovilj-Nikic," Image Quality enhancement by applying genetic algorithm in median filtering",Advances in Computer science an IT,pp.277-294.

[28] Mantas Paulinas, Andrius Ušinskas," A survey of genetic algorithms applications for image enhancement and segmentation", ISSN 1392 - 124X Information Technology and Control, 2007, Vol.36, No.3 